\title{
IRREGULAR INVARIANT MEASURES RELATED TO HAAR MEASURE
}

\author{
H. LEROY PETERSON
}

Abstract. Let $\mathrm{G}$ be a locally compact nondiscrete group, and let $\nu_{1}$ be a Haar measure on an open subgroup of $G$. It is not hard to show that $\nu_{1}$ must be the restriction of a Haar measure $\nu$ on all of $G$. Here we show that there exists a translation invariant measure $\mu$ (found by extending $\nu_{1}$ to the cosets of $H$ in a natural way) which agrees with $\nu$ on, for example, ( $\nu) \sigma$-finite sets, open sets, and subsets of $H$. Although $\nu$ can be computed from $\mu$ in a relatively simple manner, the two measures are not equal in general. In fact, there is an extreme case, namely when $H$ is not $\sigma$-compact and has uncountably many cosets, in which $\mu$ fails very badly to be regular-there are closed sets on which $\mu$ is not inner regular and (other) closed sets on which $\mu$ is not outer regular. One condition sufficient for this extreme case to be possible is when $G$ is Abelian and not $\sigma$-compact.

1. Definitions and notation. Let $\mu$ be a (nonnegative, countably additive) measure defined on a $\sigma$-algebra $M$ of subsets of a topological space $X$. If $S$ is in $M$, we say that $\mu$ is inner regular on $S$ if

$$
\mu S=\sup \{\mu C: C \in M, C \text { compact, } C \subset S\} .
$$

We say that $\mu$ is outer regular on $S$ if

$$
\mu S=\inf \{\mu U: U \in M, U \text { open, } U \supset S\} .
$$

Following $[2,11.34]$, we say that $\mu$ is regular if it is outer regular on every set in $M$ and inner regular on every open set in $M$, and if every compact set in $M$ has finite measure.

By Haar measure on a locally compact group $G$, we mean a left Haar measure as defined in, e.g., [2]; that is to say, a left-translation invariant, regular, nondegenerate measure on a $\sigma$-algebra $M(G)$ of subsets of $G . M(G)$ contains all the closed subsets of $G$ and consists of all the sets which are measurable with respect to the Carathéodory outer measure associated with the measure.

If $H$ is a subgroup of $G$ (not necessarily normal), $G / H$ denotes the space of left cosets of $H$ in $G$. If $S$ is a set, $P(S)$ denotes the collection of all subsets of $S ;|S|$ denotes the cardinality of $S$.

Received by the editors March 19, 1969 and, in revised form, July 28, 1969.

AMS Subject Classifications. Primary 2220, 2875; Secondary 2030, 2210, 2810, 2813.

Key Phrases. Locally compact group, Abelian group, translation-invariant measure, regular measure, $\sigma$-compact space. 
2. Lemma 1. Let $X$ be a Hausdorff space, $(X, M, \mu)$ a measure space, with $\mu C<\infty$ for all compact $C \in M$ and $\mu$ inner regular on $S \in M$ whenever $\mu S<\infty$. Let

$$
\begin{aligned}
\lambda S & =\sup \{\mu C: C \text { compact }, C \in M, C \subset S\}, \\
\nu S & =\inf \{\mu U: U \text { open, } U \in M, U \supset S\},
\end{aligned}
$$

for all $S \in M$. Then

(1) $\lambda S=\mu S=\nu S$ whenever $\nu S<\infty$,

(2) $\lambda$ and $\nu$ are measures.

Proof. (1) Suppose $\nu S<\infty$. Let $V$ be a $G_{\delta}$ set such that $S \subset V$ and $\nu S=\mu V$. Jf $C$ is a compact member of $M$ with $C \subset V-S$, then $S \subset$ $V-C \subset V$. Now $V-C$ is a $G_{\delta}$ set so $\mu(V-C)=\nu S=\mu V$, thus $\mu C=0$. Hence $\mu(V-S)=0$, and $\mu S=\mu V=\nu S ; \lambda S=\mu S$ is obvious since $\mu S<\infty$.

(2) Suppose $\left\{S_{j}: j=1,2, \cdots\right\} \subset M$ and $S_{j} \cap S_{k}=\varnothing(j \neq k)$. Let $S=\bigcup S_{j}$, let $C$ be a $\sigma$-compact subset of $S$ such that $\lambda S=\mu C$ and (for each $j$ ) let $C_{j}$ be a $\sigma$-compact subset of $S_{j}$ with $\mu C_{j}=\lambda S_{j}$. Then

$$
\lambda S=\mu C=\mu\left(U C \cap S_{j}\right)=\sum \mu\left(C \cap S_{j}\right) \leqq \sum \lambda S_{j}
$$

and

$$
\lambda S \geqq \mu\left(\cup C_{j}\right)=\sum \mu C_{j}=\sum \lambda S_{j},
$$

hence $\lambda$ is a measure. Clearly (by (1)),

$$
\nu S=\mu S=\sum \mu S_{j}=\sum V S_{j}
$$

if $\nu S<\infty$. If $\nu S=\infty$, take $U_{j}$ a $G_{\delta}$ set such that $U_{j} \supset S_{j}$ and $\nu S_{j}$ $=\mu U_{j}(j=1,2, \cdots)$. Then

$$
\sum \nu S_{j}=\sum \mu U_{j} \geqq \mu\left(\cup U_{j}\right) \geqq \nu S=\infty,
$$

thus $\nu$ is a measure.

LeMma 2. Let $G$ be a locally compact group, $H$ an open subgroup of $G$. Then $M(H)=M(G) \cap P(H)$.

Proof. Let $\nu$ be a Haar measure on $G$ and $\nu_{1}$ a Haar measure on $H$. Both $\nu$ and $\nu_{1}$ are unique to within a multiplicative constant; further, if $U$ is an open subset of $H$ with compact closure, then $0<\nu U<\infty$ and $0<\nu_{1} U<\infty$. Thus we may assume that $\nu U=\nu_{1} U$; but then the Carathéodory outer measures associated with $\nu$ and $\nu_{1}$, respectively, are equal on $P(H)$. It follows that the $\nu$-measurable and $\nu_{1}$-measurable subsets of $H$ coincide, which is to say that $M(H)=M(G) \cap P(H)$. 
Note. The proof of Lemma 2 contains the information that there is a one-to-one correspondence between the Haar measures on $G$ and $H$, respectively, given by $\nu \leftrightarrow \nu_{1}=\nu \mid M(H)$. One by-product of Theorem 1 will be a method of computing $\nu$, given $\nu_{1}$.

TheOREM 1. Let $G$ be a nondiscrete locally compact group, let $H$ be an open subgroup of $G$, and $\nu_{1}$ a left Haar measure on $H$. For $S \in M(G)$, define

$$
\begin{aligned}
\mu S & =\sum\left\{\nu_{1}(x S \cap H): x H \in G / H\right\} \\
\nu S & =\inf \{\mu U: U \text { open, } U \supset S\}
\end{aligned}
$$

Then

(1) $\mu$ is a well-defined left-invariant measure on $M(G)$.

(2) $\nu$ is a Haar measure for $G$.

(3) $\mu$ and $\nu$ are both extensions of $\nu_{1}$ and $\mu$ and $\nu$ agree on open sets and (v) $\sigma$-finite sets.

(4) If $H$ is not $\sigma$-compact, $\mu$ fails to be inner regular on some closed subsets of $G$.

(5) If $G / H$ is uncountable, $\mu$ fails to be outer regular on some closed subsets of $G$.

Proof. (1) We know that if $S \in M(G)$, then $x S \in M(G)$ and therefore $x S \cap H \in M(G) \cap P(H)=M(H)$ for all $x \in G$. Further, if $x H=y H$, then

$$
\nu_{1}(x S \cap H)=\nu_{1}\left(y x^{-1} x S \cap H\right)=\nu_{1}(y S \cap H),
$$

since $\nu_{1}$ is left invariant. Thus $\mu$ is well defined; it is clearly left-invariant. To show that $\mu$ is a measure, suppose $\left\{S_{j}\right\} \subset M(G), S_{j} \cap S_{k}$ $=\varnothing(j \neq k) ;$ then

$$
\begin{aligned}
\mu\left(U S_{j}\right) & =\sum \nu_{1}\left(\bigcup_{x} S_{j} \cap H\right)=\sum\left(\sum_{j=1}^{\infty} \nu_{1}\left(x S_{j} \cap H\right)\right) \\
& =\sum_{j=1}^{\infty}\left(\sum \nu_{1}\left(x S_{j} \cap H\right)\right)=\sum_{j=1}^{\infty} \mu S_{j}
\end{aligned}
$$

(by standard arguments; either both double summations have uncountably many nonzero terms or the $l_{1}$ version of Fubini's theorem applies).

(2) Let $S \in M(G)$, with $S$ open or $\mu S<\infty$. There is a countable set $\left\{x_{j}\right\}$ such that $\mu S=\sum_{j=1}^{\infty} \nu_{1}\left(x_{j} S \cap H\right)$. For each $j$, there is a $\sigma$-compact set $C_{j}$ such that $C_{j} \subset x_{j} S \cap H$ and $\nu_{1} C_{j}=\nu_{1}\left(x_{j} S \cap H\right)$. Thus

$$
\mu S=\sum \nu_{1} C_{j}=\sum \mu\left(x_{j}^{-1} C_{j}\right)=\mu\left(\bigcup_{x_{j}}^{-1} C_{j}\right) \leqq \lambda S \leqq \mu S,
$$


where $\lambda$ is as in Lemma 1 , since $U x_{j}^{-1} C_{j}$ is a $\sigma$-compact subset of $S$. Now Lemma 1 applies, so that $\nu$ is a regular measure defined on $\boldsymbol{M}(G)$; it is obvious that $\nu$ has the other properties required of a Haar measure.

(3) This statement is obvious.

(4) If $H$ is not $\sigma$-compact, then (16.14) of [2] shows that there is a closed subset of $H$ on which $\nu$ (and therefore $\mu$ ) is not inner regular.

(5) If $G / H$ is uncountable, then an argument easily derived from the proof of (16.14) (op. cit.) shows that there is a closed subset $F$ of $G$ such that $\mu F=0$ and $\nu F=\infty$; thus $\mu U=\infty$ for any neighborhood $U$ of $F$ and $\mu$ is not outer regular on $F$.

Notes on Theorem 1. I. Statements (4) and (5) each imply that $G$ is not $\sigma$-compact. Theorems 2 and 3, below, state conditions under which (4) and (5) can be true for the same subgroup $H$.

II. $\lambda$ is the inner-regular "Haar measure" described in [1, Theorem 1] (and, from a different point of view, in [4, II.1])-or, more properly, the extension of this (weakly) Borel measure to $\boldsymbol{M}(G)$.

III. For each $S$ in $M(G)$, one of the following statements must always be true:

(a) $\lambda S=\mu S=\nu S$ ( $\mu$ is outer regular and inner regular on $S$ ).

(b) $\lambda S=\mu S<\nu S=\infty$ ( $\mu$ is not outer regular on $S$ ).

(c) $\lambda S<\mu S=\nu S=\infty$ ( $\mu$ is not inner regular on $S)$.

IV. If $\nu_{1}$ were a right Haar measure, one could proceed in the same manner to obtain right-invariant measures on $\boldsymbol{M}(G)$ with the desired properties, except that right cosets of $H$ and right translates of sets would play the rôle given to left cosets and left translates in Theorem 1.

3. Lemma 3. Let $G$ be an uncountable Abelian group. Then $G$ contains a subgroup $K$ such that $|K|=|G / K|=|G|$.

Proof. Let $r=|G|$.

Case 1. Suppose $r=r_{0}(G)$, the torsion-free rank of $G$. Then there exists a maximal independent torsion-free subset $X$ of $G$ such that $|X|=r$. Let $K=\left[\left\{x^{2}: x \in X\right\}\right]$. As in [4, II.8], $|K|=|G / K|=r$.

Case 2. Suppose $G$ is torsion. Since $G$ is uncountable, it must have a subgroup $G_{1}$ of bounded order such that $\left|G_{1}\right|=r$. By (A.25) of [2], $G_{1}$ is the direct sum of cyclic groups; thus $G_{1}=[Y]$ where $Y$ is an independent set and $|Y|=r$. Let $Y=Y_{1} \cup Y_{2}$ where $Y_{1} \cap Y_{2}=\varnothing$ and $\left|Y_{1}\right|=\left|Y_{2}\right|=r ;$ let $K=\left[Y_{1}\right]$. Then $|K|=r \geqq|G / K| \geqq\left|G_{1} / K\right| \geqq\left|Y_{2}\right|$ $=r$.

Case 3. Suppose $r>r_{0}(G)$. Let $X$ be a maximal torsion-free independent set; let $G^{\prime}=G /[X] . G^{\prime}$ is a torsion group and $\left|G^{\prime}\right|=r$, so by 
Case $2 G^{\prime}$ has a subgroup $K^{\prime}$ such that $\left|K^{\prime}\right|=\left|G^{\prime} / K^{\prime}\right|=r$; let $K$ be the subgroup of $G$ such that $K^{\prime}=K /[X]$. Clearly $|K|=r$, and by the Third Isomorphism Theorem $|G / K|=\left|G^{\prime} / K^{\prime}\right|=r$.

THEOREM 2. Let $G$ be a locally compact Abelian group which is not $\sigma$-compact. Then $G$ has an open subgroup $H$ such that $H$ is not $\sigma$-compact and $G / H$ is uncountable.

Proof. Let $U$ be an open $\sigma$-compact subgroup of $G$; then $G^{\prime}=G / U$ is uncountable and by Lemma 3 has a subgroup $K^{\prime}$ such that $\left|K^{\prime}\right|$ $=\left|G^{\prime} / K^{\prime}\right|=\left|G^{\prime}\right|$. Let $H$ be the subgroup of $G$ such that $K^{\prime}=H / U$. Then $H$ is not $\sigma$-compact since $H / U$ is a cover of $H$ by uncountably many pairwise disjoint open sets. Also, $|G / H|=\left|G^{\prime} / K^{\prime}\right|=\left|G^{\prime}\right|$.

THEOREM 3. Let $G$ be a locally compact group which is not the union of fewer than $\boldsymbol{\aleph}_{2}$ compact sets. Then $G$ has an open subgroup $H$ such that $H$ is not $\sigma$-compact and $G / H$ is uncountable.

Proof. Let $U$ be a $\sigma$-compact open subgroup of $G$ and let $H$ be a subgroup of $G$ generated by a collection of $\boldsymbol{\aleph}_{1}$ cosets of $U$. $H$ has the desired properties.

4. Examples (the group $R_{d} \times R$ ). Let $G$ be the group $R_{d} \times R$, where $R_{d}$ is the discrete reals and $R$ is the reals with the usual topology. Let $\lambda_{0}$ be Lebesgue measure on $R$, and for $r \in R_{d}$, let $\lambda_{r}(S)$ $=\lambda_{0}(\{x:(r, x) \in S\})$. Define

$$
\lambda S=\sum\left\{\lambda_{r}(S): r \in R_{d}\right\} .
$$

Case 1. (From [3, \$12.58]). Let $H=\{0\} \times R$ and $\nu_{1}=\lambda \mid \boldsymbol{M}(H)$ $=$ Lebesgue measure on $\{0\} \times R$. Here $H$ is $\sigma$-compact and $G / H$ is uncountable, being isomorphic to $R_{d}$. We have $\lambda=\mu$, and $\mu F_{1}=0$, $\nu F_{1}=\infty$, where $F_{1}=R_{d} \times\{0\}$.

Case 2. Let $K$ be the subgroup of $R_{d}$ generated by a Hamel basis over $Q$; let $H=K \times R$. In this case, $H$ is not $\sigma$-compact and $G / H$ is uncountable. For $S \in M(G)$, we have

$$
\begin{aligned}
\nu S & =\inf \{\lambda U: U \text { open, } U \supset S\}, \\
\nu_{1} & =\nu \mid M(H),
\end{aligned}
$$

as natural choices for Haar measures. Here,

$$
\mu S=\sum\left\{\nu(S \cap(\{r\} \times R)): r \in K_{2}\right\}
$$

where $K_{2}$ is a subgroup of $R_{d}$ such that $R_{d}=K_{2} \oplus K$. Let $F_{2}=K_{2} \times\{0\}$; then $\lambda F_{1}=0, \mu F_{1}=\nu F_{1}=\infty$ and $\lambda F_{2}=\mu F_{2}=0, \nu F_{2}=\infty$. 


\section{REFERENCES}

1. S. K. Berberian, Counterexamples in Haar measure, Amer. Math. Monthly 73 (1966), 135-140.

2. E. Hewitt and K. A. Ross, Abstract harmonic analysis. I: Structures of topological groups. Integration theory. Group representations, Academic Press, New York and Springer-Verlag, Berlin, 1963. MR 28 \#158.

3. E. Hewitt and K. R. Stromberg, Real and abstract analysis. A modern treatment of the theory of functions of a real variable, Springer-Verlag, New York, 1965. MR 32 \#5826.

4. H. L. Peterson, Regular and irregular measures on groups and dyadic spaces, Pacific J. Math. 28 (1969), 173-182.

University of Connecticut 